\title{
Effect of oral insulin on insulin autoantibody levels in the Diabetes Prevention Trial Type 1 oral insulin study
}

\author{
J. M. Barker • K. K. McFann • T. Orban • \\ on behalf of DPT-1 Study Group
}

Received: 16 January 2007 / Accepted: 19 March 2007 / Published online: 22 June 2007

(C) Springer-Verlag 2007

\begin{abstract}
Aims/hypothesis Our aim was to evaluate insulin autoantibody (IAA) levels over time in the Diabetes Prevention Trial Type 1 (DPT-1) oral insulin study to determine the effect of oral insulin compared with placebo on IAA levels. Subjects and methods The DPT-1 trial randomised 372 relatives of subjects with type 1 diabetes, positive for IAA and with normal IVGTTs and OGTTs, to oral insulin $7.5 \mathrm{mg}$ daily or placebo. Subjects were followed with IVGTTs, OGTTs and serial IAA measurements. The change in IAA level over time was modelled statistically using mixed model longitudinal data analysis with spatial exponential law for unevenly spaced data. In a separate analysis, subjects were divided into four groups by treatment and diabetes status at the end of the study. IAA levels were compared amongst the groups at randomisation, last sampling and at the maximum level.

Results Longitudinal data analysis showed that treatment did not affect levels of IAA over time. After controlling for age, the IAA levels at randomisation and the last visit and
\end{abstract}

Electronic supplementary material The online version of this article (doi:10.1007/s00125-007-0694-0) contains supplementary material, including a list of the members of the DPT-1 Study Group. This is available to authorised users.

J. M. Barker · K. K. McFann

Barbara Davis Center for Childhood Diabetes,

?University of Colorado Health Sciences Center,

Denver, CO, USA

T. Orban

Joslin Diabetes Center,

Boston, MA, USA

J. M. Barker $(\bowtie)$

Barbara Davis Center for Childhood Diabetes,

Box A140, Building M20, 1775 N. Ursula Street, P.O. Box 6511,

Aurora, CO 80045-6511, USA

e-mail: jennifer.barker@uchsc.edu the maximum values were different in the four groups. Significantly higher levels were noted in groups that developed diabetes compared with those that did not, with no significant difference by treatment group.

Conclusions/interpretation This suggests that IAA levels over time were not influenced by oral insulin in subjects already positive for IAA at the start of treatment.

\section{ClinicalTrials.gov ID no.: NCT00004984.}

Keywords Insulin autoantibodies · Oral insulin . Prevention · Type 1 diabetes

$\begin{array}{ll}\text { Abbreviations } \\ \text { DPT-1 } & \text { Diabetes Prevention Trial Type } 1 \\ \text { IAA } & \text { insulin autoantibodies } \\ \text { ICA } & \text { islet cell autoantibody }\end{array}$

\section{Introduction}

The Diabetes Prevention Trial Type 1 (DPT-1) oral insulin study randomised subjects to oral insulin or placebo. While the oral insulin therapy showed no delay in diabetes, posthoc analysis suggested that in subjects with high insulin autoantibodies (IAA) treatment with oral insulin resulted in a delay in diabetes of approximately 4 years [1]. Therefore, further trials of oral insulin are planned. It is known that s.c. insulin injections induce insulin antibodies [2]. However, the effect of oral insulin on IAA is not known. The oral insulin arm of the DPT-1 provides the ideal cohort in which to evaluate the change in IAA levels in response to treatment with oral insulin.

We hypothesised that subjects who received oral insulin and those who developed diabetes would have higher levels 
of IAA compared with the placebo group and those who did not develop diabetes, respectively. To test this hypothesis, we compared IAA levels over time in subjects who received and did not receive insulin and those who developed and did not develop diabetes in the DPT- 1 oral insulin study.

\section{Subjects and methods}

DPT-1 recruited first- and second-degree relatives of people with type 1 diabetes for a study of the natural history of type 1 diabetes and oral and parenteral insulin diabetes prevention trials. Detailed descriptions of the trials and results have been previously published $[1,3]$. Briefly, relatives were screened for islet cell autoantibodies (ICA). Those positive for ICA were tested for IAA, IVGTTs and OGTTs and HLA haplotypes associated with protection from diabetes (HLA $D Q A^{*} 0102, D Q B^{*} 0602$ ). Subjects positive for ICA and IAA with normal IVGTTs and OGTTs and without the protective HLA haplotype were invited to participate in the oral insulin diabetes prevention study. Subjects were randomised to either oral insulin $(7.5 \mathrm{mg}$ daily recombinant human insulin crystals (Eli Lilly, Indianapolis, IN, USA)) or placebo and were followed every 6 months with an OGTT and IAA determination. Data analysed in this report were obtained from the DPT-1 de-identified database release December 2004 (revised October 2005).

IAA assay IAA was measured in a standard RIA incorporating competition with unlabelled insulin. Human ${ }^{125}$ I-labelled insulin $(20,000 \mathrm{cpm})$ was incubated with serum for 7 days at $4{ }^{\circ} \mathrm{C}$, precipitated with polyethylene glycol and counted in a gamma counter [4]. The activity of radiolabelled insulin was $37 \mathrm{kBq}$. The cut-off for entry into the study was initially $\geq 80 \mathrm{nU} / \mathrm{ml}$ and was decreased to $\geq 40 \mathrm{nU} / \mathrm{ml}$ [1]. In the 2005 Diabetes Antibody Standardization Program for proficiency testing, this assay with a cut-off point of $40 \mathrm{nU} / \mathrm{ml}$ had $46 \%$ sensitivity and $96 \%$ specificity.

Statistical analysis In this analysis, subjects were divided into four groups: group 1, subjects receiving oral insulin who developed diabetes by the end of the study; group 2, subjects receiving oral insulin who did not develop diabetes by the end of the study; group 3, subjects receiving oral placebo who developed diabetes; and group 4, subjects receiving oral placebo who did not develop diabetes.

Statistical analyses were performed using SAS 9.1 for Windows (SAS, Cary, NC, USA). The groups were compared for demographic characteristics including age at randomisation and diabetes, duration of follow-up, sex and number of IAA measurements. IAA levels at randomisation, the maximum IAA level reached and the last IAA level were evaluated. As the distribution of IAA values is not normal, the levels were logarithmically transformed for analysis $(\ln \mathrm{IAA}=\ln [\mathrm{IAA}+61])$. In order to facilitate interpretation of results, the results were back-transformed. Analysis of covariance was performed to compare logarithmically transformed IAA levels at randomisation, the maximum level and the level at the last visit across the four groups adjusting for age. A Tukey-Kramer correction to test for significant differences between groups was employed.

The change in IAA level over time was modelled statistically using mixed model longitudinal data analysis with a spatial exponential low for unevenly spaced data [5]. All available data points from each patient were used for this analysis. Variables entered into the model included treatment (oral insulin or placebo) and duration of observation (study) and an interaction variable between the two.

Table 1 IAA levels (nU/ml) in the DPT-1 oral insulin trial

\begin{tabular}{|c|c|c|c|c|c|c|}
\hline & $R^{2}$ & $p$ value & $\begin{array}{l}\text { Group } 1 \text { (oral insulin, } \\
\text { diabetes) } n=45\end{array}$ & $\begin{array}{l}\text { Group } 2 \text { (oral insulin, } \\
\text { no diabetes) } n=141\end{array}$ & $\begin{array}{l}\text { Group } 3 \text { (oral placebo, } \\
\text { diabetes) } n=53\end{array}$ & $\begin{array}{l}\text { Group } 4 \text { (oral placebo, } \\
\text { no diabetes) } n=133\end{array}$ \\
\hline ANCOVA & 0.13 & $<0.0001$ & & & & \\
\hline Randomisation IAA & & 0.0032 & $258(183-355)$ & $236(194-285)$ & $333(245-447)$ & $182(151-218)^{\mathrm{a}}$ \\
\hline Age & & $<0.0001$ & & & & \\
\hline ANCOVA & 0.16 & $<0.0001$ & & & & \\
\hline Last IAA & & $<0.0001$ & $258(154-412)$ & $136(109-167)$ & $363(235-545)^{\mathrm{b}}$ & $110(87-137)^{\mathrm{a}, \mathrm{c}}$ \\
\hline Age & & $<0.0001$ & & & & \\
\hline ANCOVA & 0.16 & $<0.0001$ & & & & \\
\hline Maximum IAA & & $<0.0001$ & $530(360-769)$ & $367(306-439)$ & $725(518-1007)^{\mathrm{b}}$ & $290(245-342)^{\mathrm{a}, \mathrm{c}}$ \\
\hline Age & & $<0.0001$ & & & & \\
\hline
\end{tabular}

Results are shown as back-transformed means $(95 \% \mathrm{CI})$

ANCOVA, analysis of covariance

${ }^{\text {a }}$ Significantly different from group 3

${ }^{\mathrm{b}}$ Significantly different from group 2

${ }^{\mathrm{c}}$ Significantly different from group 1 
This analysis allows for data collection times to vary among subjects while taking into account the correlation of repeated measurements on each subject. A second model incorporating observations where BMI, GAD65 and ICA512 autoantibody levels were available in addition to the variables in the original model was analysed.

\section{Results}

Three hundred and forty subjects were first degree relatives of a person with type 1 diabetes (91\%) and 263 (71\%) had a confirmed IAA level $\geq 80 \mathrm{nU} / \mathrm{ml}$. There were no differences in the groups based upon age at randomisation, sex distribution, age at diabetes or number of IAA tests performed. Subjects in group 2 were followed for a longer period of time than subjects in groups 1 and $3(p=0.002)$ (Electronic supplementary material [ESM] Table 1).

IAA levels at randomisation and the last visit and the maximum IAA levels are shown in Table 1. After controlling for age, IAA levels at randomisation differed according to progression to diabetes only in the placebo group (groups 3 and 4). There were no differences in the last and/or maximum IAA levels according to active or placebo treatment in either those who progressed to diabetes (groups 1 and 3) or those who remained nondiabetic (groups 2 and 4). Figure 1 shows all IAA values for each subject divided into groups. IAA levels across the entire range of values throughout the study are seen in all groups.

The mixed model longitudinal analysis showed no significant effect of oral insulin vs placebo over time on IAA level. However, there was a small but statistically significant effect of time on study and results showed that IAA tended to decrease as study time increased. Younger age was associated with a higher IAA level (ESM Table 2). Subanalysis of 343 subjects with 1,524 observations including the above variables, BMI, GAD65 and ICA-512 levels was also performed and showed no influence of BMI, GAD65 and ICA-512 on IAA over time but a
Fig. 1 IAA levels over time in the DPT-1 oral insulin trial by treatment group and diabetes status. a Oral insulin diabetes (group 1); b oral insulin no diabetes (group 2); c placebo diabetes (group 3); d placebo no diabetes (group 4). Dotted lines represent low $(\geq 40 \mathrm{nU} / \mathrm{ml})$ and high ( $\geq 80 \mathrm{nU} / \mathrm{ml})$ cut-offs for positivity
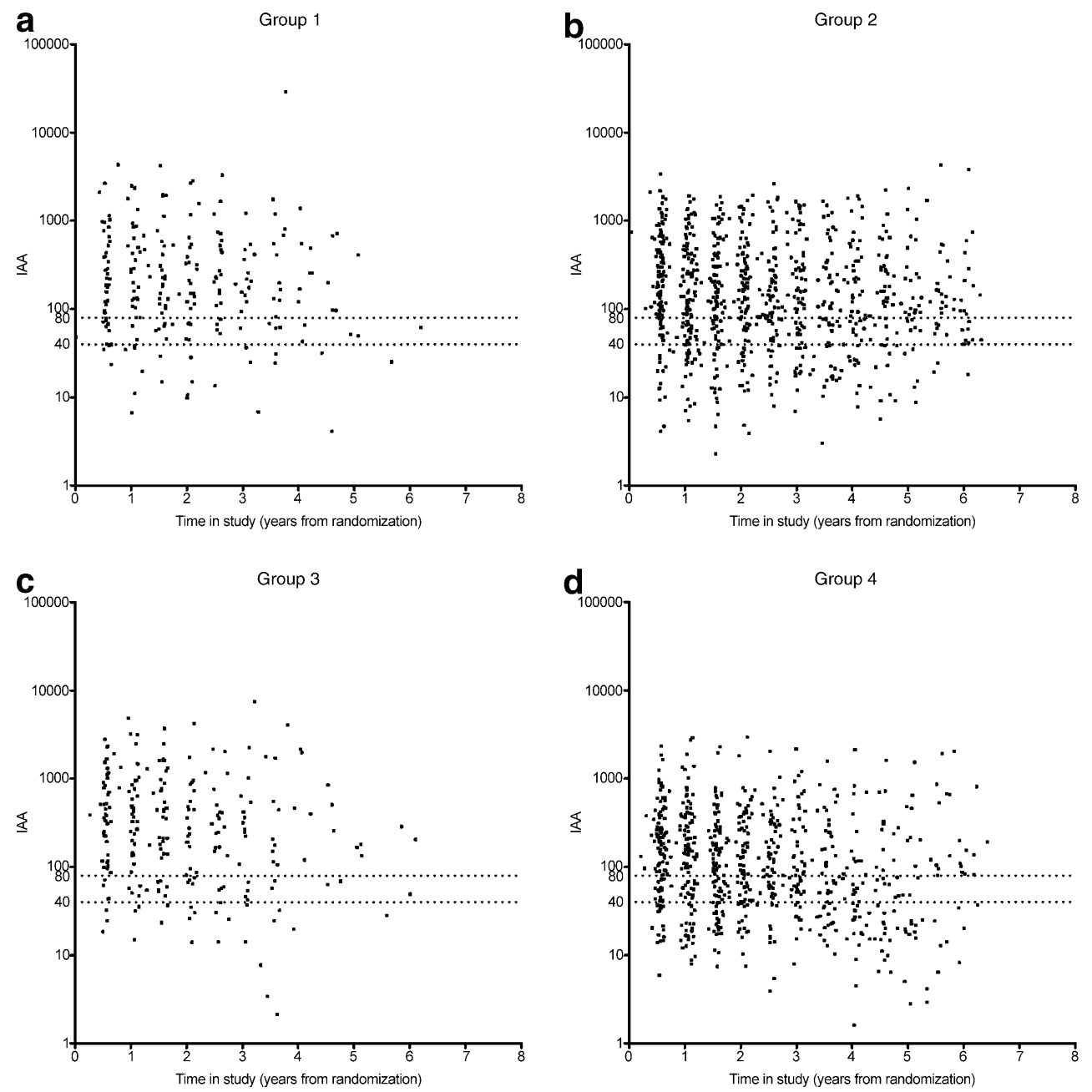
significant effect of age at randomisation and time on study on IAA levels. When analysis was limited to subjects with IAA $>80 \mathrm{nU} / \mathrm{ml}$, there was no effect of therapy on IAA levels over time. There was no interaction between treatment group and time on study.

\section{Discussion}

Our analysis of IAA levels in DPT-1 showed that subjects who eventually developed diabetes had higher baseline and peak IAA levels compared with subjects who had not developed diabetes by the end of the DPT-1 study. However, there was not a significant difference in IAA levels between oral insulin- and placebo-treated subjects in the groups that did not develop diabetes, suggesting that IAA levels were not influenced by treatment with oral insulin at a dose of $7.5 \mathrm{mg}$ daily. Autoantibody level is only one aspect of the autoimmune response. Factors such as IgG subtype [6, 7], IAA affinity [8, 9] and T cell responses to insulin [10] are also important markers of the immune response to insulin. Therefore, by evaluating only IAA levels we may be missing the immunological effect of oral insulin. Trials of oral insulin in groups at risk of type 1 diabetes are planned. Evaluation of IAA affinity, autoantibody subtype and $\mathrm{T}$ cell response to insulin to identify an immunological response will be important components of these trials.

These data have some limitations for generalisation to populations considered for future prevention trials. The assay in this differs from the assay currently used for monitoring insulin autoantibodies, the micro-IAA assay. Subjects who participated in the oral insulin arm of DPT-1 were all positive for IAA at the time of randomisation, making it impossible to assess the effect of oral insulin on IAA for antibody-negative subjects. Additionally, subjects in the DPT-1 cohort are all relatives of subjects with type 1 diabetes. There is no reason to suspect that subjects from the general population would respond differently to oral insulin, but this was not specifically tested. It is important to point out that the choice of a $7.5 \mathrm{mg}$ dose was somewhat arbitrary and it is conceivable that different doses of oral insulin may have different effects on IAA levels.

In conclusion, analysis of IAA levels in the DPT- 1 oral insulin study suggests that IAA levels in subjects positive for
IAA at the initiation of oral insulin at $7.5 \mathrm{mg}$ daily do not show an increase compared with those of placebo-treated. The effect of oral insulin on the immune system may be masked by the presence of IAA at onset of therapy, or there may not be a significant effect on the immune system when administered at this dose. Further evaluations including $\mathrm{T}$ cell assays will be important in characterising the immune response to oral insulin in future prevention trials.

Acknowledgements The authors thank the subjects and their families who participated in the DPT-1, without whom none of this would be possible. J. M. Barker is supported by a grant from the Juvenile Diabetes Research Foundation (11-2005-15). We also wish to acknowledge support of CRC NIH M01 RR00069.

Duality of interest The authors report no duality of interest

\section{References}

1. Diabetes Prevention Trial Type 1 Study Group (2005) Effects of oral insulin in relatives of patients with type 1 diabetes: the Diabetes Prevention Trial-Type 1. Diabetes Care 28:1068-1076

2. Lauritano AA, Clements RS Jr, Bell D (1989) Insulin antibodies in non-insulin-dependent diabetes mellitus: effect of treatment with semisynthetic human insulin. Clin Ther 11:268-277

3. Diabetes Prevention Trial Type 1 Study Group (2002) Effects of insulin in relatives of patients with type 1 diabetes mellitus. N Engl J Med 346:1685-1691

4. Vardi P, Dib SA, Tuttleman M et al (1987) Competitive insulin autoantibody assay. Prospective evaluation of subjects at high risk for development of type 1 diabetes mellitus. Diabetes 36:1286-1291

5. Jones RH (1993) Longitudinal data with serial correlation: a statespace approach. Monogr Stat Appl Probab 47:25-76

6. Achenbach P, Warncke K, Reiter J et al (2004) Stratification of type 1 diabetes risk on the basis of islet autoantibody characteristics. Diabetes 53:384-392

7. Hoppu S, Ronkainen MS, Kimpimaki T et al (2004) Insulin autoantibody isotypes during the prediabetic process in young children with increased genetic risk of type 1 diabetes. Pediatr Res 55:236-242

8. Achenbach P, Koczwara K, Knopff A, Naserke H, Ziegler AG, Bonifacio E (2004) Mature high-affinity immune responses to (pro)insulin anticipate the autoimmune cascade that leads to type 1 diabetes. J Clin Invest 114:589-597

9. Achenbach P, Warncke K, Reiter J et al (2006) Type 1 diabetes risk assessment: improvement by follow-up measurements in young islet autoantibody-positive relatives. Diabetologia 49:2969-2976

10. van de Linde P, Roep BO (2005) T-cell assays to determine disease activity and clinical efficacy of immune therapy in type 1 diabetes. Am J Ther 12:573-579 